\title{
Outcome of Severe Traumatic Brain Injury at KSMC: Functional Outcomes of ICP Monitor Insertion-Two Years' Experience
}

This article was published in the following Dove Press journal: Open Access Emergency Medicine

\author{
Abdul Raman M AL-Shudifat ${ }^{1,2}$ \\ Babar Kahlon ${ }^{2}$ \\ Sarah Bin Abdulqader ${ }^{2}$ \\ Wajab Almutairi ${ }^{2}$ \\ Khairiah Alsumali ${ }^{2}$ \\ Yazeed Aldhfyan ${ }^{2}$ \\ Laith Al-Abdallat (D) \\ 'Department of Neurosurgery, School of \\ Medicine, The University of Jordan, \\ Amman, Jordan; ${ }^{2}$ Department of \\ Neurosurgery, King Saud Medical City \\ (KSMC), Riyadh, Saudi Arabia
}

\begin{abstract}
Purpose: To evaluate functional outcomes of severe traumatic brain injuries after insertion of intracranial pressure (ICP) monitor at King Saud Medical City (KSMC) and their
\end{abstract} correlation to each other.

Patients and Methods: A retrospective observational study for all adult patients (age $>18$ years) who were diagnosed with severe head injury and underwent ICP insertion at KSMC. Patients diagnosed between 2017 and 2019 were included. Data for measured outcomes, Glasgow outcome scale (GOS), Karnofsky Performance Score (KPS) and length of stay (LOS) and prognostic factors, data like: age, gender and primary Glasgow coma score (GCS) was obtained from patients' files and direct communication with patients or their caregivers. We also compared patients who underwent ICP monitoring alone with those who underwent ICP with decompressive craniectomy (DC). Follow-up period ranged from 6-24 months.

Results: Seventy-four patients were included in this cohort study. Outcome measurements for patients with decompression and ICP were lower than those with ICP alone. KPS and GOS showed strong correlation $(p<0.01)$ in whole cohort and in both subgroups (ICP alone and ICP with DC). KPS showed significant correlation with length of stay ( $p=0.026)$.

Conclusion: ICP monitoring is valid tool in management of severely head injured patients. Patients who underwent DC had a worse outcome. KPS can be used as alternative tool to measure functional outcome in severe traumatic brain injury.

Keywords: traumatic brain injury, functional outcome, ICP monitoring, decompressive craniectomy

\section{Introduction}

Traumatic brain injury (TBI) is considered as one of the most common causes of mortality in the young population. Motor vehicle accidents (MVA) are considered the main cause of severe traumatic brain injury in developing countries. ${ }^{7}$ A TBI can result in a wide spectrum of clinical picture depending on the severity, that might affect cognition, mobility, sensation, behavior, and speech. ${ }^{5,21}$ In addition, it has long-term sequelae like financial burden and social impact both on affected people and caregivers. ${ }^{14}$

Management of TBI depends on the severity of the injury. Many guidelines were described in the literature regarding management and can help in improving outcomes, with Brain Trauma Foundation (BTF) guidelines being the worldwideadopted measures for severe head injury management. ${ }^{6}$ Intracranial pressure (ICP) monitoring is the mainstay and steering factor in these guidelines, where clear
Correspondence: Laith Al-Abdallat Department of Neurosurgery, School of Medicine, The University of Jordan, Queen Rania Street, Amman, I 1942, Jordan

Tel +962797333219

Email abdallat97@outlook.com 
indications were established for ICP insertion. Neuroimaging and clinical examination are still used in some centers to monitor patients with severe head injury. ${ }^{8}$

The evaluation of these established guidelines need validated outcome measurements and tools. Mortality rate, neurological outcomes, hospital stay, in-hospital complications, quality of life, and functional outcome measurements like the Glasgow Outcome Scale (GOS) are currently used as outcome measurements for severe head injury management. ${ }^{15,17}$ TBI is considered a major concern in our region with significant impact on health resources and unwanted consequences on social life. ${ }^{11}$ Therefore, in this study we aim to evaluate functional outcomes after insertion of ICP for patients who suffered from severe traumatic brain injury at KSMC, the biggest referral trauma center in Saudi Arabia.

\section{Patients and Methods}

Approval was obtained from Institutional Review Board at King Saud Medical Complex, approval number H1R124Jun19-02. Study was conducted in accordance with the Declaration of Helsinki. Informed consent was obtained prior to study commencement from the patient himself/ herself, or from a relative when patient had low GCS.

Data for patients and measured outcomes (GOS, KPS, 30-day mortality rate and length of stay) for those who underwent ICP monitoring over two years at KSMC were obtained from patients' files and direct contact with caregivers or patients themselves. The Codman Neuro device was used for intraparenchymal ICP monitoring, with continuous monitoring and recording under ICU staff control. Prognostic factors (age, gender, and primary GCS) were obtained from patients' files. Furthermore, we divided our cohort into two subgroups: patients who underwent ICP monitoring alone and those underwent ICP with decompressive craniectomy (DC). Follow-up period was 6-24 months.

\section{Statistical Analysis}

Inclusion criteria: all patients who diagnosed with severe traumatic brain injury (GCS <8) between January 2017 and January 2019 and underwent ICP insertion at our hospital. Exclusion criteria include patients with missed follow-up after discharge. Our criteria for ICP insertion are in concordance with TBI foundation guidelines with TBI protocol for management of head injury. ${ }^{6}$

Data for patients and measured outcomes was obtained from patients' files and direct contact with caregivers or patients themselves.
We used $p$-value significance and Pearson's correlation between different variables, and we did not go further in multivariable because of the results in univariate analysis. Software used was SPSS.

\section{Results}

\section{Study Population}

A total of 74 patients fulfilled the inclusion criteria after exclusion of 14 patients with missed data, 47 patients were treated by ICP insertion and 27 patients treated by ICP insertion followed by decompressive craniectomy. Details of patients and injury characteristics were illustrated in Table 1. Mean age was 26.6 years $( \pm 14.7)$. Males $(88 \%)$ were more affected than females $(12 \%)$. MVA constituted the majority of admitted cases $(85.1 \%)$. There was no statistically significant difference between both subgroups in age, gender and GCS at admission.

\section{Outcome Measurements}

All outcome measurements for the group who underwent ICP and DC were worse than those for the ICP group alone, but without statistically significant difference, see Table 1. Although its value was high (89 days), length of stay for the ICP and DC group did not show significant difference between both subgroups. Age and gender also tested for possible correlation with outcome measurements, which revealed no statistical correlation (data not shown).

\section{Correlation Between Outcome Measurements}

By using Pearson's correlation, we tested outcome measurements (GOS, KPS, length of stay) against each other for the whole group and both subgroups for possible correlation. Interestingly, KPS was strongly correlated to GOS in all groups $(p<0.01)$. Moreover, in the ICP subgroup without decompression, KPS showed significant correlation with length of stay $(p=0.026)$, see Table 2 .

\section{Discussion}

Severe TBI is a major burden, affecting 10 million people per year worldwide. ${ }^{18,24}$ In Saudi Arabia most patients $(56.7 \%)$ had severe TBI (GCS 3-8). ${ }^{3}$ Patients admitted with severe TBI constituted $(93.2 \%)$ of the total mortality rate, MVC was the main mechanism of $\mathrm{TBI}^{2-4}$ Our cohort, which was restricted to severe traumatic head 
Table I Study Population and Subgroups Characteristics

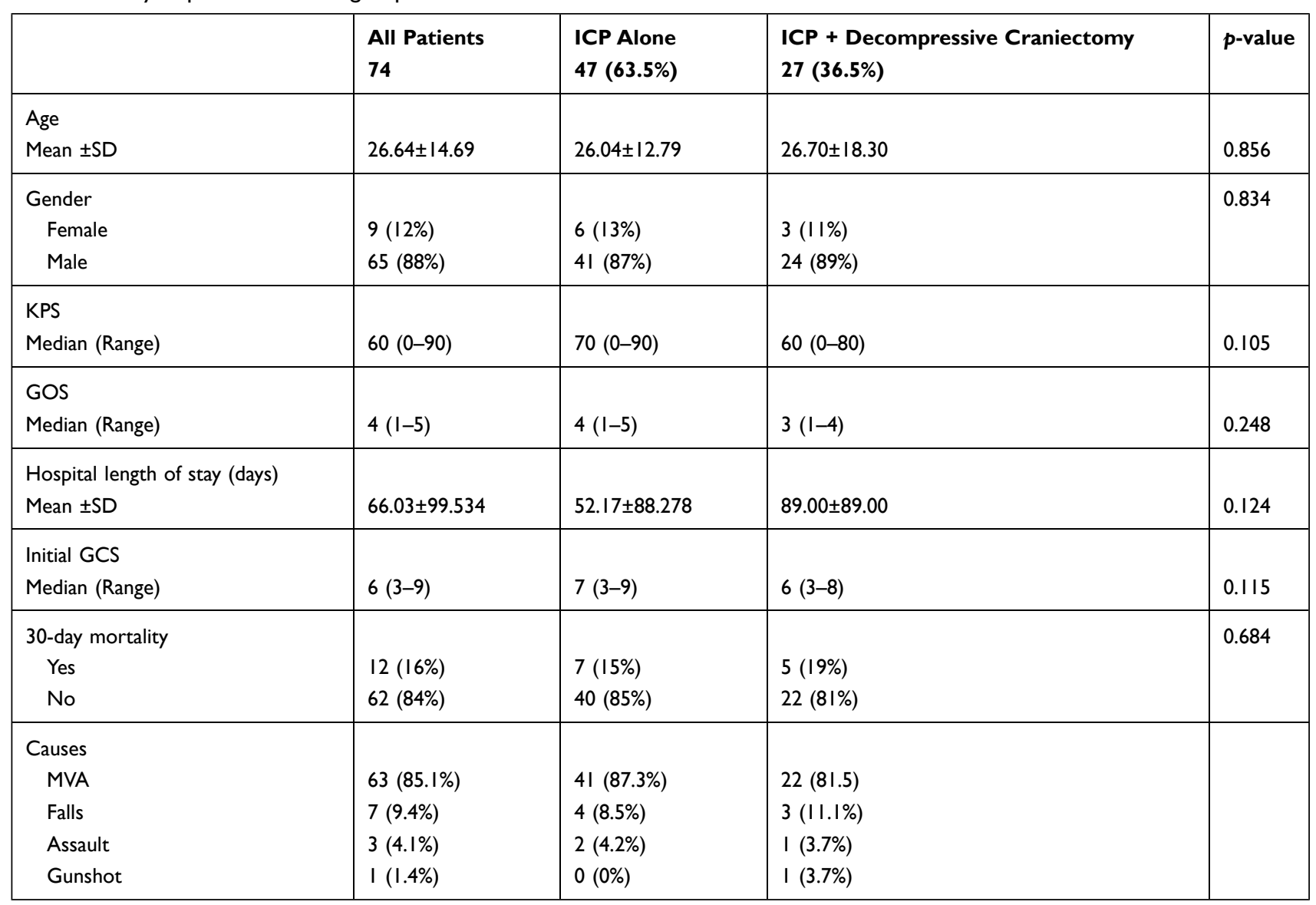

Table 2 Pearson's Correlation Between Outcome Measurements (Whole Group, ICP Group and, ICP with Decompressive Craniectomy)

\begin{tabular}{|c|c|c|c|}
\hline & GOS & KPS & Length of Stay \\
\hline \multicolumn{4}{|l|}{ Whole group } \\
\hline GOS & - & $0.0006 *$ & 0.144 \\
\hline KPS & $0.0006^{*}$ & - & 0.085 \\
\hline Length of stay & 0.144 & 0.085 & - \\
\hline \multicolumn{4}{|l|}{ ICP group } \\
\hline GOS & - & $0.0006 *$ & 0.055 \\
\hline KPS & $0.0006^{*}$ & - & $0.026 *$ \\
\hline Length of stay & 0.055 & $0.026 *$ & - \\
\hline \multicolumn{4}{|l|}{ ICP and DC group } \\
\hline GOS & - & $0.0008^{*}$ & 0.801 \\
\hline KPS & $0.0008^{*}$ & - & 0.786 \\
\hline Length of stay & 0.801 & 0.786 & - \\
\hline
\end{tabular}

Note: *Statistical significance $(p<0.05)$.

injury in the adult population treated in our center, showed that MVA is the most common cause for severe traumatic brain injury (85.1\%) with young male patients as the most affected group.
Different management protocols for severe TBI were discussed in the literature. Most of these protocols use ICP monitor insertion as a guide for management, as it also proved to improve primary outcome. ${ }^{1,8,9}$ Although decompressive craniectomy was described as part of the treatment when there is refractory medical treatment for brain edema secondary to trauma, many reports were doubting its efficacy and its role in outcome improvement. ${ }^{17,20,22}$ In our center we have adopted most of the Brain Trauma Foundation (BTF) guidelines of management of severe traumatic brain injury where ICP monitor insertion and decompressive craniectomy indications were clearly stated in our protocol for many years. Our current study demonstrated, in comparison with previous literature, ${ }^{17,19}$ acceptable outcome values (KPS, GOS, length of stay) for the whole group and subgroups see Table 1. As expected, the subgroup that underwent decompressive craniectomy with ICP monitor insertion showed lower outcome values, ${ }^{16}$ owing to their low initial GCS secondary to severe primary impact injury.

Neurological deficits, mortality, hospital stay, quality of life, and functional outcome were used as instruments to 
measure outcome of severe TBI. ${ }^{6,22}$ Functional outcome like GOS, disability indexes, and return to work were used variably in the literature to compare different treatment protocols for severe traumatic brain injury in adults. ${ }^{10,23}$ Many predicting factors were mentioned in these reports that could affect outcome like: age, initial GCS, ICP readings, etc. In our cohort, studying these factors was beyond the scope of this study, but we examined correlation of age and initial GCS with measured outcomes and they were not statistically significant. In addition, we found no statistically significant difference between both subgroups (ICP alone and ICP with DC) in age, gender and initial GCS, which made comparison of outcomes between these subgroups more reasonable.

This study is one of the few studies on patients with severe TBI that used KPS as a primary outcome measurement. ${ }^{13,17,25}$ To our knowledge, most of functional outcomes were focused on GOS or its extended version (GOSE), though it has many drawbacks and critiques like crudeness, narrow scale, and no clear structured administration rules. ${ }^{12}$ KPS has more precise assessment of functional level with a wider score range. Our results showed strong correlation between GOS and KPS in the whole group and both subgroups with significant correlation between KPS and length of stay in the group with ICP monitor insertion alone, while GOS did not show any correlation with length of stay in any group.

This study has many limitations like retrospective design, relatively small sample size, variable follow-up period, and limited confounders studied. However, we think that we presented our experience at one of the biggest trauma centers in Saudi Arabia focusing on functional outcome of severe traumatic brain injury in adults may trigger further investigation in this field. Furthermore, we believe that using KPS as an instrument to measure functional outcome may result in more sensitive and accurate assessment of functional level than the GOS. Consequently, KPS may be a valuable alternative to the GOS for assessing functional outcome after severe TBI in adults.

\section{Conclusion}

Severe traumatic brain injury has unwarranted effects on measured functional outcomes. ICP monitoring is a valid tool in management of severe traumatic brain injury. Patients who underwent decompressive craniectomy had a worse outcome than those treated with ICP alone. KPS can be used as an alternative tool to measure functional outcome in severe traumatic brain injury. Larger and prospective studies are recommended to test the validity of use of such a tool in evaluation of functional outcome of severe traumatic brain injury in adults.

\section{Disclosure}

Our abstract has been presented at a conference (SANS,2020-Riyadh) as a poster but not in a previously published article. Abdul Raman M AL-Shudifat, Babar Kahlon, Sarah Bin Abdulqader, Wajab Almutairi, Khairiah Alsumali, Yazeed Aldhfyan, and Laith AlAbdallat report no other conflicts of interest in this work.

\section{References}

1. Aiolfi A, Benjamin E, Khor D, Inaba K, Lam L, Demetriades D. Brain trauma foundation guidelines for intracranial pressure monitoring: compliance and effect on outcome. World $J$ Surg. 2017;41:1543-1549. doi:10.1007/s00268-017-3898-6

2. Alhabdan S, Zamakhshary M, Al Naimi M, et al. Epidemiology of traumatic head injury in children and adolescents in a major trauma center in Saudi Arabia: implications for injury prevention. Ann Saudi Med. 2013;33(1):52-56. doi:10.5144/0256-4947.2013.52

3. Al-Habib A, A-shail A, Alaqeel A, et al. Causes and patterns of adult traumatic head injuries in Saudi Arabia: implications for injury prevention. Ann Saudi Med. 2013;33(4):351-355. doi:10.5144/02564947.2013.351

4. Alnaami I, Alshehri S, Alghamdi S, et al. Patterns, types, and outcomes of head injury in Aseer Region, Kingdom of Saudi Arabia. Neurosci j. 2019;2019:1-6. doi:10.1155/2019/2782146

5. Bhalerao SU, Geurtjens C, Thomas GR, Kitamura CR, Zhou C, Marlborough M. Understanding the neuropsychiatric consequences associated with significant traumatic brain injury. Brain Inj. 2013;27 (7-8):767-774. doi:10.3109/02699052.2013.793396

6. Carney N, Totten AM, O'Reilly $\mathrm{C}$, et al. Guidelines for the Management of Severe Traumatic Brain Injury. 4th ed. 2017. doi:10.1227/NEU.0000000000001432

7. Bratton SL, Chestnut RM, Ghajar J, et al. Guidelines for the management of severe traumatic brain injury. Indications for intracranial pressure monitoring. $J$ Neurotrauma. 2007;24:S37-S44. doi:10.1089/neu.2007.9990

8. Chesnut RM, Temkin N, Carney N, et al. Global Neurotrauma Research Group: a trial of intracranial-pressure monitoring in traumatic brain injury. $N$ Engl $J$ Med. 2012;367(26):2471-2481. doi:10.1056/NEJMoa1207363

9. Chesnut RM, Bleck TP, Citerio G, et al. A consensus-based interpretation of the benchmark evidence from South American trials: treatment of intracranial pressure trial. J Neurotrauma. 2015;32 (22):1722-1817. doi:10.1089/neu.2015.3976

10. Cifu DX, Kreutzer JS, Marwitz JH, Rosenthal M, Englander J, High W. Functional outcomes of older adults with traumatic brain injury: a prospective, multicenter analysis. Arch Phys Med Rehabil. 1996;77(9):883-888. doi:10.1016/S0003-9993(96)90274-9

11. DeSilva MJ, Roberts I, Perel P, et al. Patient outcome after traumatic brain injury in high-, middle- and low-income countries: analysis of data on 8927 patients in 46 countries. Int J Epidemiol. 2009;38 (2):452-458. doi:10.1093/ije/dyn 189

12. Dikmen S, Machamer J, Manley GT, Yuh EL, Nelson LD, Temkin NR. Functional status examination versus glasgow outcome scale extended as outcome measures in traumatic brain injuries: how do they compare? J Neurotrauma. 2019;36(16):2423-2429. doi:10.1089/neu.2018.6198 
13. Elhusseiny H, Elzamly K, Leon K, Nobleza CO. The role of palliative care in adult moderate to severe traumatic brain injury. Palliat Med Care. 2018;5(3):1-11. doi:10.15226/2374-8362/5/3/00159

14. Finkelstein EA, Corso PS, Miller TR. The Incidence and Economic Burden of Injuries in the United States. New York: Oxford University Press; 2006.

15. Gupta D, Sharma D, Kannan N. Guideline adherence and outcomes in severe adult traumatic brain injury for the CHIRAG (Collaborative Head Injury and Guidelines) Study. World Neurosurg. 2016;89 (89):169-179. doi:10.1016/j.wneu.2015.12.097

16. Hutchinson PJ, Kolias AG, Timofeev IS, et al. Trial of decompressive craniectomy for traumatic intracranial hypertension. $N$ Engl J Med. 2016;375:1119-1130. doi:10.1056/NEJMoa1605215

17. Khan F, Valliani A, Rehman A, Bari ME. Factors affecting functional outcome after decompressive craniectomy performed for traumatic brain injury: a retrospective, cross-sectional study. Asian $J$ Neurosurg. 2018;13(3):730-736. doi:10.4103/ajns.AJNS_6_18

18. Langlois JA, Rutland-Brown W, Wald MM. The epidemiology and impact of traumatic brain injury a brief overview. J Head Trauma Rehabil. 2006;21(5):375-378. doi:10.1097/00001199-20060900000001

19. Maas AI, Roozenbeek B, Manley GT. Clinical trials in traumatic brain injury: past experience and current developments. Neurotherapeutics. 2010;7:115-126. doi:10.1016/j.nurt.2009.10.022
20. Sahuquillo J, Arikan F. Decompressive craniectomy for the treatment of refractory high intracranial pressure in traumatic brain injury. Cochrane Database Syst Rev. 2006;2006. doi:10.1002/14651858. CD003983.pub2

21. Semple BD, Zamani A, Rayner G, Shultz SR, Jones NC. Affective, neurocognitive and psychosocial disorders associated with traumatic brain injury and post-traumatic epilepsy. Neurobiol Dis. 2019;123:27-34.

22. Timofeev I, Kirkpatrick PJ, Corteen E, et al. Decompressive craniectomy in traumatic brain injury: outcome following protocol-driven therapy. Acta Neurochir Suppl. 2006;96:11-16.

23. Utomo WK, Gabbe BJ, Simpson PM, Cameron PA. Predictors of in-hospital mortality and 6-month functional outcomes in older adults after moderate to severe traumatic brain injury. Injury. 2009;40 (9):973-977. doi:10.1016/j.injury.2009.05.034

24. Walder B, Haller G, Rebetez MM, et al. Severe traumatic brain injury in a high-income coun- try: an epidemiological study. J Neurotrauma. 2013;30(23):1934-1942. doi:10.1089/neu.2013.2955

25. Zhao J, Chen H, Zhang M, et al. Early expression of serum neutrophil gelatinase-associated lipocalin (NGAL) is associated with neurological severity immediately after traumatic brain injury. J Neurol Sci. 2016;368:392-398. doi:10.1016/j.jns.2016.07.060

\section{Publish your work in this journal}

The Open Access Emergency Medicine is an international, peerreviewed, open access journal publishing original research, reports, editorials, reviews and commentaries on all aspects of emergency medicine. The manuscript management system is completely online and includes a very quick and fair peer-review system, which is all easy to use. Visit http://www.dovepress.com/testimonials.php to read real quotes from published authors. 